\title{
Detailed phase matching characterization of inter-modal four-wave mixing in a two-mode fiber
}

\author{
S. M. M. Friis ${ }^{1,2}$, Y. Jung ${ }^{1}$, I. Begleris ${ }^{1}$, P. Horak ${ }^{1}$, K. Rottwitt ${ }^{2}$, P. Petropoulos ${ }^{1}$, \\ D. J. Richardson ${ }^{1}$, and F. Parmigiani ${ }^{1}$ \\ ${ }^{1}$ Optoelectronics Research Centre, University of Southampton, SO17 1BJ, United Kingdom \\ ${ }^{2}$ Department of Photonics Engineering, Technical University of Denmark, 2800 Kongens Lyngby, Denmark \\ smmf@fotonik.dtu.dk; frp@orc.soton.ac.uk
}

\begin{abstract}
We experimentally characterize the phase matching properties of two inter-modal four-wave mixing processes in a graded index fiber guiding the LP01 and LP11 mode-groups.
\end{abstract}

OCIS codes: 060.2270 Fiber characterization; 190.4380 Nonlinear optics, four-wave mixing.

\section{Introduction}

Back in the 1970's, Stolen et al. [1] observed four-wave mixing (FWM) among waves of different frequencies occupying distinct spatial modes in a few tens of meters of multi-mode fiber. Recently, Essiambre et al. [2] have experimentally observed two non-degenerate inter-modal FWM processes, phase conjugation (PC) and Bragg scattering (BS) [3], through several kilometers of few-mode fiber with low differential group velocity between the modes. An extensive theoretical study was conducted by Xiao et al. [4] that highlighted the difference in the performance obtained in PC and BS through phase matching, mainly in terms of signal bandwidth.

In this paper, we report experimental characterizations of the aforementioned inter-modal FWM processes in terms of generated idler power and bandwidth of the phase matching in a $1 \mathrm{~km}$ graded index fiber. We show that the bandwidth depends greatly on the wavelength separation of the two pumps and that the bandwidth of BS is broader than that of PC at the optimum pump separation, in good agreement with the theory.

\section{Experimental results}

Our experimental setup is shown in Fig. 1. Three continuous wave tunable laser sources (TLSs) are used as the two pumps and the signal in the BS and the PC processes. To avoid stimulated Brillouin scattering and increase the peak power, all three sources are gated with a $10 \%$ duty cycle at a repetition rate of $10 \mathrm{MHz}$. One pump (p1) and the signal (s) waves are launched into the LP01 mode while the second pump (p2) wave is launched into the LP11 mode using a mode-multiplexer (MMUX) based on a phase plate (PP) to achieve the PC and BS processes, respectively, see Fig. 2(a) and (b). Polarization controllers and a polarization beam splitter (PBS) guarantee that the interacting waves are co-polarized. Two Erbium-doped fiber amplifiers (EDFAs) are used to achieve $20.5 \mathrm{dBm}$ average power in each pump at the input of the fiber under test (FUT). The FUT is a $1 \mathrm{~km}$ graded index few-mode fiber supporting mode-groups

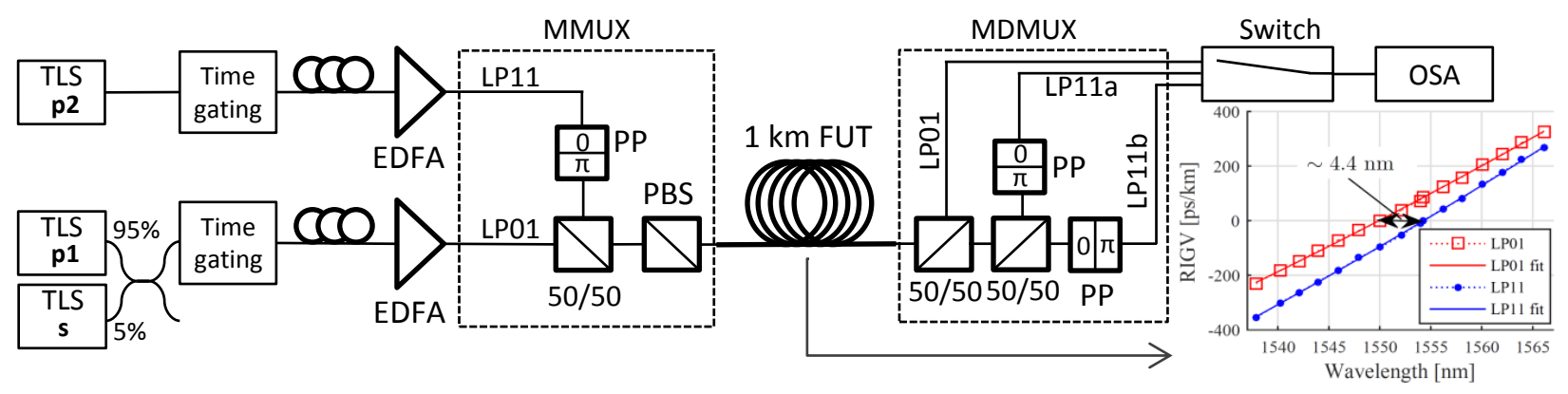

Fig. 1. Experimental setup. 50/50 denotes a non-polarization beam splitter. The inset shows measured relative inverse group velocities (RIGVs) of the LP01 and LP11 mode-groups. 

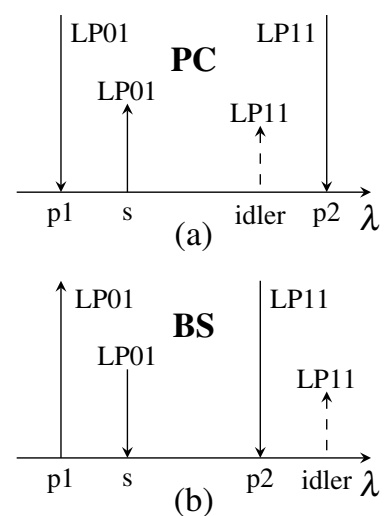

(b)
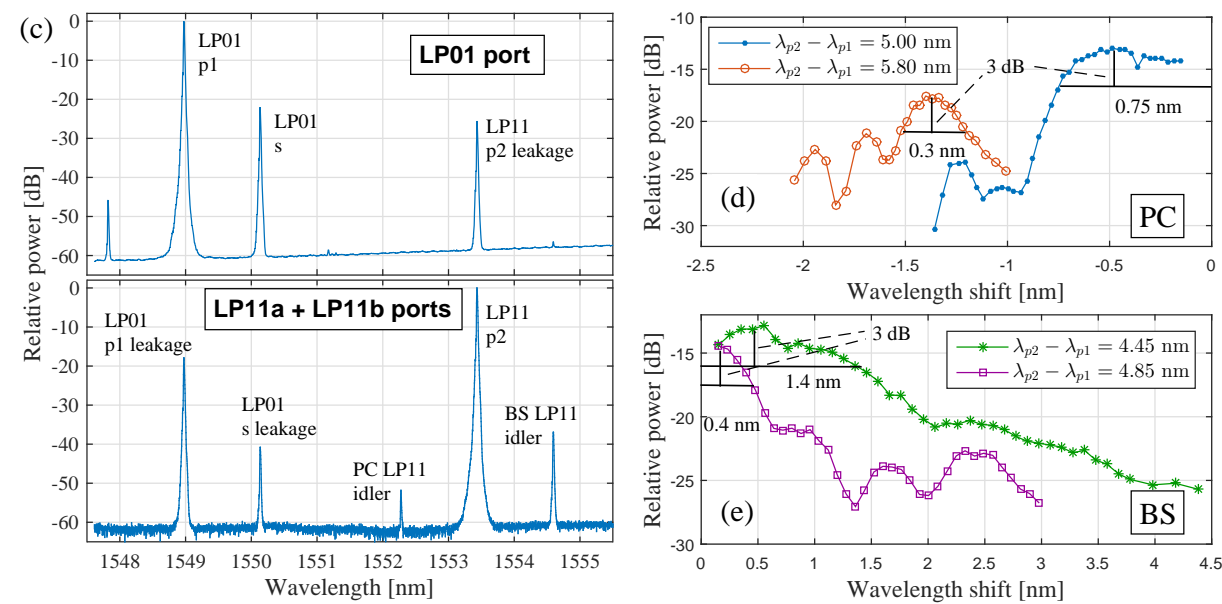

Fig. 2. (a) and (b) wavelength configurations of $\mathrm{PC}$ and BS, respectively; the arrows indicate gaining (up) and losing (down) power; (c) MDMUX spectra for $\lambda_{p 2}=1553.45 \mathrm{~nm}$ and $\lambda_{s}=1550.15 \mathrm{~nm}$; (d) and (e) idler power relative to signal power vs. idler wavelength shift for PC and BS, respectively.

LP01 and LP11; the effective areas of the LP01 and LP11 modes are $161 \mu \mathrm{m}^{2}$ and $170 \mu \mathrm{m}^{2}$ and their dispersion coefficients are $D^{(01)}=19.8 \mathrm{ps} /(\mathrm{km} \mathrm{nm})$ and $D^{(11)}=21.8 \mathrm{ps} /(\mathrm{km} \mathrm{nm})$ at $1550 \mathrm{~nm}$, respectively. The inset of Fig. 1 shows the measured RIGV of the LP01 and LP11 modes; the waves in the two modes propagate at the same group velocity at a wavelength separation of about $4.4 \mathrm{~nm}$ at $1550 \mathrm{~nm}$. At the output of the FUT, we mode-demultiplex (MDMUX) the LP01, LP11a and LP11b by coupling them into single-mode fibers (SMFs): in the LP01 detection port, the LP11 mode-group cannot propagate into the SMF, and in the LP11 detection ports, two orthogonally orientated phase plates are used to collect the LP11a and LP11b, respectively, while simultaneously phase-shifting the LP01 such that it is not guided by the SMF. A switch enables quick sampling of all three ports in the optical spectrum analyzer (OSA).

Figure 2(c) shows typical LP01 port output (top) and combined LP11a and LP11b ports output (bottom) spectra; each port of the MDMUX extinguishes the other mode-group with an efficiency of approx. $20 \mathrm{~dB}$. Figure 2(d) and (e) show the LP11 idler power relative to the LP01 signal power for PC and BS, respectively, versus the wavelength shift of the generated idler relative to $\mathrm{p} 2$, achieved by varying the signal wavelength. The two curves in each plot represent different wavelength separations between the pumps; the wavelength of $\mathrm{p} 1$ is $\lambda_{p 1}=1549.00 \mathrm{~nm}$ and the wavelength shifts of $\mathrm{p} 2$ are shown in the legends. Theoretical investigations predict the broadest signal bandwidth for both PC and BS when the wavelength separation between the pumps is close to the separation of the RIGV curves in Fig. 1, i.e. about $4.4 \mathrm{~nm}$, and the bandwidth gets narrower (with shifts in the central peaks) as the wavelength separation between the pumps changes, with more severe changes for the BS. Our results confirm these trends: for PC, the maximum bandwidth of $0.75 \mathrm{~nm}$ at a pump separation of $\lambda_{p 2}-\lambda_{p 1}=5.00 \mathrm{~nm}$ decreases to $0.3 \mathrm{~nm}$ at $\lambda_{p 2}-\lambda_{p 1}=5.8 \mathrm{~nm}$, i.e. the bandwidth reduces by a factor 2.5 when the wavelength of $\mathrm{p} 2$ is changed $0.8 \mathrm{~nm}$, and the peak of maximum idler power is moved about $0.9 \mathrm{~nm}$; for BS, the maximum bandwidth of $1.4 \mathrm{~nm}$ at $\lambda_{p 2}-\lambda_{p 1}=4.45 \mathrm{~nm}$ decreases to $0.4 \mathrm{~nm}$ at $\lambda_{p 2}-\lambda_{p 1}=4.85 \mathrm{~nm}$, i.e. the bandwidth reduces by a factor of 3.5 when the wavelength of $\mathrm{p} 2$ is changed by only $0.4 \mathrm{~nm}$. Note that when the signal and p1 are close in wavelength, other FWM processes interfere with PC and BS, both intra- and inter-modal, which complicates evaluating the bandwidth of phase matching.

In conclusion, we experimentally characterized the phase matching properties of two inter-modal four-wave mixing processes, phase conjugation and Bragg scattering. We measured the generated LP11 idler output power vs. LP01 signal wavelength for the two processes and their corresponding bandwidths for various pump wavelength separations.

\section{References}

1. R. H. Stolen et al., Appl. Phys. Lett. 24, 308-310 (1974).

2. R.-J. Essiambre et al., IEEE Photon. Technol. Lett. 25, 539-542 (2013).

3. C. J. McKinstrie et al., IEEE J. Sel. Top. Quantum Electron. 18, 958-969 (2012).

4. Y. Xiao et al., Opt. Express, 22, 32039-32059 (2014). 